\title{
BIRKHOFF NORMAL FORMS, KAM THEORY, PERIODICITY AND SYMMETRIES FOR CERTAIN RATIONAL DIFFERENCE EQUATION WITH CUBIC TERMS
}

\author{
MEHMED NURKANOVIĆ AND ZEHRA NURKANOVIĆ \\ Dedicated to the memory of Professor Mahmut Bajraktarević
}

\begin{abstract}
By using the Kolmogorov-Arnold-Moser (KAM) theory, we investigate the stability of the positive elliptic equilibrium point of the difference equation

$$
x_{n+1}=\frac{A x_{n}^{3}+B}{a x_{n-1}}, \quad n=0,1,2, \ldots
$$

where the parameters $A, B, a$ and the initial conditions $x_{-1}, x_{0}$ are positive numbers. The specific feature of this difference equation is the fact that we were not able to use the invariant to prove stability or to find feasible periods of the solutions.
\end{abstract}

\section{INTRODUCTION AND PRELIMINARIES}

We consider the dynamics of the following equation

$$
x_{n+1}=\frac{A x_{n}^{3}+B}{a x_{n-1}}, \quad n=0,1, \ldots
$$

where the parameters $A, B, a$ and the initial conditions $x_{-1}, x_{0}$ are positive numbers. Equation (1) is a special case of the more general equation

$$
x_{n+1}=\frac{A x_{n}^{k}+B}{a x_{n-1}}, \quad n=0,1, \ldots,
$$

where $k$ is a fixed number of the set $\{1,2,3, \ldots\}$. Note that equation (2) has very interesting special cases as Lyness' equation (for $k=1, A=a=1$, see

2010 Mathematics Subject Classification. 37E40, 37J40, 37N25, 39A10, 39A11.

Key words and phrases. Area preserving map, Birkhoff normal form, KAM theorem, stability, twist coefficient.

Copyright (c) 2016 by ANUBIH. 
$[9,11,18])$ or the equation

$$
x_{n+1}=\frac{A x_{n}^{2}+F}{e x_{n-1}}, \quad n=0,1, \ldots
$$

which was studied in [7]. These equations were considered by either the Kolmogorov-Arnold-Moser (KAM) theory or combination of algebraic and geometric techniques. The second technique was always based on the existence of invariants, see $[10,9,8,12,13,14]$. However, but in the case of the equation (3) it was not possible to find an invariant of this equation which generates the area preserving map and the only available technique seems to be the KAM theory. It seems that equation (1) is of this type as we were not able to find any invariant. Also, the simulations and visualizations of the orbits of this equation do not indicate the existence of some invariant (see Figure 1). Note that the paper [1] gives some effective tests for difference equation to have a continuous invariant.

In this paper we will show that in equation (2) there exist the same situations with the number equilibrium points and their stability properties as in equation (1). Also, we will show that equation (1) is more complicated than equation (3). As we can see in [7], equation (3) either has no positive equilibrium points or has a unique positive equilibrium, which is non-hyperbolic of the elliptic type and which is stable. On the other hand, for equation (1) we have three qualitative distinct situations: the equation has no positive equilibrium points, or has a unique positive equilibrium point which is nonhyperbolic of parabolic type, or has two positive equilibrium points: one of these is a saddle and the other is non-hyperbolic of the elliptic type.

Indeed, by substituting

$$
x_{n}=\sqrt{\frac{B}{a}} w_{n}
$$

in (1) we obtain

$$
w_{n+1}=\frac{\frac{A}{a} \sqrt{\frac{B}{a}} w_{n}^{3}+1}{w_{n-1}},
$$

i.e. $\left(\right.$ for $\left.\alpha=\frac{A}{a} \sqrt{\frac{B}{a}}\right)$

$$
w_{n+1}=\frac{\alpha w_{n}^{3}+1}{w_{n-1}} .
$$

The equilibrium points of equation (4) are the positive solutions of the equation

or equivalently

$$
\bar{w}=\frac{\alpha \bar{w}^{3}+1}{\bar{w}}
$$

$$
\alpha \bar{w}^{3}-\bar{w}^{2}+1=0 .
$$


Set $g(w)=\alpha w^{3}-w^{2}+1$. It is obvious that the function $g$ has the local maximum $g_{\max }=1$ for $w=0$ and has a local minimum for $w=\frac{2}{3 \alpha}$. Also, we see that equation (5):

a) has no positive roots if $g\left(\frac{2}{3 \alpha}\right)>0$, or equivalently if $\alpha>\frac{2}{3 \sqrt{3}}$,

b) has only one positive root if $g\left(\frac{2}{3 \alpha}\right)=0$, or equivalently if $\alpha=\frac{2}{3 \sqrt{3}}$,

c) has two positive roots if $g\left(\frac{2}{3 \alpha}\right)<0$, or equivalently if $0<\alpha<\frac{2}{3 \sqrt{3}}$.

This means that equation (4):

$\mathrm{a}_{1}$ ) has no positive equilibrium points for $\alpha>\frac{2}{3 \sqrt{3}}$,

$\mathrm{b}_{1}$ ) has only one positive equilibrium point $E=E_{1}=E_{2}=\sqrt{3}$ for $\alpha=\frac{2}{3 \sqrt{3}}$,

$\left.\mathrm{c}_{1}\right)$ has two positive equilibrium points $E_{1}$ and $E_{2}\left(1<E_{1}<\frac{2}{3 \alpha}\right.$ and $\left.E_{2}>\frac{2}{3 \alpha}>\sqrt{3}\right)$ for $0<\alpha<\frac{2}{3 \sqrt{3}}$.

We will show that the equilibrium point $E=E_{1}$ for $0<\alpha<\frac{2}{3 \sqrt{3}}$ is an elliptic point of an area preserving map since eigenvalues of the $J_{T}\left(E_{1}\right)$ form a purely imaginary, complex conjugate pair $\lambda, \bar{\lambda}$, see $[11,6]$. This means that equation (4) has very specific dynamics and that the KAM theory is the appropriate tool to investigate the dynamics of the equation since we will prove that the map $T$ corresponding to equation (4) is an area preserving map.

An area preserving map $T$ is a map that preserves the area of any measurable planar region $S \subset \mathbb{R}^{2}$ under the forward iterates of the map, that is, the area of $T(S)$ equals the area of $S$. The formal definition is as follows, see [11].

Definition 1. A map $T: \mathbb{R}^{2} \rightarrow \mathbb{R}^{2}$ is said to be area preserving if its Jacobian matrix $J_{T}$ satisfies

$$
\operatorname{det} J_{T}(\mathbf{x})=1 \text { at every point } \mathbf{x} \text { of domain of } T \text {. }
$$

The stability of the elliptic type equilibrium point is established by simplifying the nonlinear terms trough appropriate coordinate transformations and bringing them into so-called normal forms. One well-known normal form is Birkhoff normal form, see [11].

Now, we present some the basic results about Birkhoff normal forms and the KAM theory. The next result gives normal form of an equation with an elliptic fixed point, see $[5,6,11,15,17]$.

Theorem 1. (Birkhoff Normal Form) Let $T: \mathbb{R}^{2} \rightarrow \mathbb{R}^{2}$ be an area-preserving $C^{n}$ map (n-times continuously differentiable) with a fixed point at the origin whose complex-conjugate eigenvalues $\lambda$ and $\bar{\lambda}$ are on the unit disk (elliptic fixed point). Suppose there exists an integer $l$ such that

$$
4 \leq l \leq n+1
$$


and suppose that the eigenvalues satisfy

$$
\lambda^{k} \neq 1 \text { for } k=3,4, \ldots, l \text {. }
$$

Let $r=\left[\frac{l}{2}\right]$ be the integer part of $\frac{l}{2}$. Then there exists a smooth function $g(z, \bar{z})$ that vanishes with its derivatives up to order $r-1$ at $z=0$, and there exists a real polynomial

$$
\alpha(\omega)=\alpha_{1} \omega+\alpha_{2} \omega^{2}+\cdots+\alpha_{r} \omega^{r}
$$

such that the map $T$ can be reduced to the normal form by suitable change of complex coordinates

$$
z \rightarrow T(z, \bar{z})=\lambda z e^{i \alpha(z \bar{z})}+g(z, \bar{z})
$$

In other words the corresponding system of difference equations

$$
x_{n+1}=T\left(x_{n}\right)
$$

can be reduced to the form

$$
\left(\begin{array}{c}
s_{n+1} \\
t_{n+1}
\end{array}\right)=\left(\begin{array}{cc}
\cos \omega & -\sin \omega \\
\sin \omega & \cos \omega
\end{array}\right)\left(\begin{array}{c}
s_{n} \\
t_{n}
\end{array}\right)+\left(\begin{array}{c}
O_{l} \\
O_{l}
\end{array}\right)
$$

where

$$
\omega=\sum_{k=0}^{M} \gamma_{k}\left(s_{n}^{2}+t_{n}^{2}\right)^{k}, \quad M=\left[\frac{l}{2}\right]-1 .
$$

Here $O_{l}$ denotes a convergent power series in $s_{n}$ and $t_{n}$ with terms of order greater than or equal to $l$ which vanishes at the origin and $[x]$ denotes the least integer greater than or equal to $x$.

The numbers $\gamma_{1}, \ldots, \gamma_{k}$ are called twist coefficients. Using Theorem 1 we can state the main stability result for an elliptic fixed point, known as the the KAM Theorem (or Kolmogorov-Arnold-Moser Theorem), see [5, 6, 11, 17].

Theorem 2. (KAM Theorem) Let $T: \mathbb{R}^{2} \rightarrow \mathbb{R}^{2}$ be an area-preserving map with an elliptic fixed point at the origin satisfying the conditions of Theorem 1. If the polynomial $\alpha\left(|z|^{2}\right)$ is not identically zero, then the origin is a stable equilibrium point. In other words if for some $k \in\{1, \ldots, M\}$ we have $\gamma_{k} \neq 0$ in (7), then the origin is a stable equilibrium point.

Remark 1. ([7]) Consider an invariant annulus $A_{\varepsilon}=\{z: \varepsilon<|z|<2 \varepsilon\}$ in a neighborhood of the elliptic fixed point, for $\varepsilon$ a sufficiently small positive number. Note that the linear part of normal form approximation leaves invariant all circles. The motion restricted to each of these circles is a rotation by some angle, see Theorem 2.28 in [11]. Also note that if at least one of the twist coefficients $\gamma_{k}$ is non-zero, the angle of rotation will vary from circle to circle. A radial line through the fixed point will undergo twisting under the mapping. The KAM theorem says that, under the addition of 
the remainder term, most of these invariant circles will survive as invariant closed curves under the original map $[5,6,11]$. Precisely, the following result holds, see $[5,6,11]$.

Theorem 3. Assuming that $\alpha(z \bar{z})$ is not identically zero and $\varepsilon$ is sufficiently small, then the map $T$ has a set of invariant closed curves of positive Lebesgue measure close to the original invariant circles. Moreover, the relative measure of the set of surviving invariant curves approaches full measure as $\varepsilon$ approaches 0 . The surviving invariant closed curves are filled with dense irrational orbits.

The following is a consequence of Moser's twist map theorem [5, 6, 17].

Theorem 4. Let $T: \mathbb{R}^{2} \rightarrow \mathbb{R}^{2}$ be an area-preserving diffeomorphism, and $(\bar{x}, \bar{y})$ a nondegenerate elliptic fixed point. There exist periodic points with arbitrarily large period in every neighborhood of $(\bar{x}, \bar{y})$.

Indeed, Theorem 3 implies that arbitrarily close to the fixed point there are always infinitely many gaps between consecutive invariant curves that contain periodic points. Within these gaps, one finds, in general, orbits of hyperbolic and elliptic periodic points. These facts cannot be deduced from computer pictures. The linearized part of (6) represent a rotation for angle $\omega$ and so if $\omega$ is rational multiple of $\pi$ every solution is periodic with same period while if $\omega$ is irrational multiple of $\pi$ there will exist chaotic solutions. In this paper we will not go into detailed study of these behaviors, as we were not able to find any continuous invariant for equation (4).

\section{KAM THEORY APPLIED TO EQUATION (4) FOR $\alpha \in\left(0, \frac{2}{3 \sqrt{3}}\right)$}

In this section, using KAM theorem, we will prove that the equilibrium point $E=E_{1}$ for $0<\alpha<\frac{2}{3 \sqrt{3}}$ is stable and that there exist an infinite number of periodic solutions.

\section{Theorem 5.}

i) If $\alpha=\frac{2}{3 \sqrt{3}}$, then equation (4) has a unique positive equilibrium point $E_{n h}=\sqrt{3}$ which is non-hyperbolic of the parabolic type.

ii) If $\alpha \in\left(0, \frac{2}{3 \sqrt{3}}\right)$, then equation (4) has two positive equilibrium points:

a) $E=E_{1}, 1<E<\frac{2}{3 \alpha}$, which is non-hyperbolic of the elliptic type and which is stable,

b) $E_{2}>\frac{2}{3 \alpha}>\sqrt{3}$, which is a saddle. 
Proof. For equilibrium point $E$ of equation (4) and for $0<\alpha<\frac{2}{3 \sqrt{3}}$ we use the substitution

$$
\begin{aligned}
& x_{n}=\ln \frac{w_{n}}{E}, \\
& y_{n}=x_{n-1},
\end{aligned}
$$

to transform equation(4) into the system

$$
\left.\begin{array}{rl}
x_{n+1} & =-y_{n}+\ln \left(\alpha E^{3} e^{3 x_{n}}+1\right)-2 \ln E \\
y_{n+1} & =x_{n} .
\end{array}\right\} \quad n=0,1,2, \ldots .
$$

Then the equilibrium point $E$ is transformed into $(0,0)$.

The map $T$ corresponding to the system (8) is of the form

$$
T\left(\begin{array}{l}
x \\
y
\end{array}\right)=\left(\begin{array}{c}
-y+\ln \left(\alpha E^{3} e^{3 x}+1\right)-2 \ln E \\
x
\end{array}\right),
$$

and the Jacobian matrix of the map $T$ at the point $(x, y)$ has the form

$$
J_{T}(x, y)=\left(\begin{array}{cc}
\frac{3 \alpha E^{3} e^{3 x}}{\alpha E^{3} e^{3 x}+1} & -1 \\
1 & 0
\end{array}\right) .
$$

It is easy to see that

$$
\operatorname{det} J_{T}(x, y)=1
$$

i.e. the map $T$ is an area preserving map. It means that we can apply KAM theory to the system (8).

Notice that

$$
J_{0}=J_{T}(0,0)=\left(\begin{array}{cc}
3 \alpha E & -1 \\
1 & 0
\end{array}\right) .
$$

The characteristic equation at $(0,0)$ is

$$
\lambda^{2}-3 \alpha E \lambda+1=0
$$

with the characteristic roots

$$
\lambda=\frac{3 \alpha E+\sqrt{9 \alpha^{2} E^{2}-4}}{2}, \quad \bar{\lambda}=\frac{3 \alpha E-\sqrt{9 \alpha^{2} E^{2}-4}}{2} .
$$

For the equilibrium $E=E_{1}=E_{2}=\sqrt{3}$, when is $\alpha=\frac{2}{3 \sqrt{3}}$, we have that $\lambda_{1}=\lambda_{2}=1$ ( $E$ is non-hyperbolic equilibrium of parabolic type). In this case we can not apply the KAM theory. If $0<\alpha<\frac{2}{3 \sqrt{3}}$, then for the equilibrium $E=E_{1}<E_{2}$ we have that $E_{1}<\frac{2}{3 \alpha}$, which implies $4-9 \alpha^{2} E_{2}^{2}>0$ and

$$
\lambda\left(E_{2}\right)=\frac{3 \alpha E_{2}+i \sqrt{4-9 \alpha^{2} E_{2}^{2}}}{2}, \quad \bar{\lambda}\left(E_{2}\right)=\frac{3 \alpha E_{2}-i \sqrt{4-9 \alpha^{2} E_{2}^{2}}}{2} .
$$

It means that $E$ is non-hyperbolic equilibrium of elliptic type and in this case we can apply the KAM theory. 
Similarly, for the equilibrium $E_{2}$ we have that $E_{2}>\frac{2}{3 \alpha}$, which implies $4-9 \alpha^{2} E_{3}^{2}<0$ and

$$
\begin{aligned}
& \lambda\left(E_{3}\right)=\frac{3 \alpha E_{3}+\sqrt{9 \alpha^{2} E_{3}^{2}-4}}{2}>\frac{3 \alpha E_{3}}{2}>1, \\
& \bar{\lambda}\left(E_{3}\right)=\frac{3 \alpha E_{3}-\sqrt{9 \alpha^{2} E_{3}^{2}-4}}{2}<\frac{3 \alpha E_{3}}{2}<1,
\end{aligned}
$$

and so the equilibrium $E_{2}$ is a saddle point.

Now, we apply KAM theory for the equilibrium $E=E_{1}, E_{1}<\frac{2}{3 \alpha}, \alpha \in$ $\left(0, \frac{2}{3 \sqrt{3}}\right)$.

Clearly $|\lambda(E)|=1,(\lambda(E))^{3} \neq 1,(\lambda(E))^{4} \neq 1$ for $\alpha \in\left(0, \frac{2}{3 \sqrt{3}}\right)$. Indeed,

$$
\begin{aligned}
& \lambda^{2}=\frac{9}{2} \alpha^{2} E^{2}-1+\frac{3}{2} i \alpha E \sqrt{4-9 \alpha^{2} E^{2}}, \\
& \lambda^{3}=\frac{9}{2} \alpha E\left(3 \alpha^{2} E^{2}-1\right)+\frac{1}{2} i(3 \alpha E-1)(3 \alpha E+1) \sqrt{4-9 \alpha^{2} E^{2}}, \\
& \lambda^{4}=\frac{1}{2}\left(81 \alpha^{4} E^{4}-36 \alpha^{2} E^{2}+2\right)+\frac{3}{2} \alpha E\left(9 \alpha^{2} E^{2}-2\right) i \sqrt{4-9 \alpha^{2} E^{2}} .
\end{aligned}
$$

If $3 \alpha E-1=0$, then it is clear that $\lambda^{3}=-1$, and similarly, if $9 \alpha^{2} E^{2}-2=0$, then $\lambda^{4}=-1$.

Thus the assumptions of Theorem 1 are satisfied for $l=4$ and we will find the Birkhoff normal form of (8) by using the sequence of transformations described in Section 1.

\section{First transformation:}

Notice that the matrix of the linearized system at the origin is the matrix

$$
J_{0}=\left(\begin{array}{cc}
3 \alpha E & -1 \\
1 & 0
\end{array}\right)
$$

and the matrix of the corresponding eigenvectors which correspond to $\lambda$ and $\bar{\lambda}$ of $J_{0}$ is

$$
P=\left(\begin{array}{cc}
\frac{1}{\lambda} & 1 \\
\lambda & \lambda
\end{array}\right)
$$

In order to obtain the Birkhoff normal form of system (8) we will expand the right hand sides of the equations of system (8) at the equilibrium point $(0,0)$ up to the order $l-1=3$ and so we obtain

$$
\begin{aligned}
& x_{n+1}=\frac{3(E-1)(E+1)}{E^{2}} x_{n}-y_{n}+\frac{9(E-1)(E+1)}{2 E^{4}}\left(x_{n}^{2}+\frac{2-E^{2}}{E^{2}} x_{n}^{3}\right)+O_{4}, \\
& y_{n+1}=x_{n} .
\end{aligned}
$$


The change of variables

$$
\left[\begin{array}{l}
x_{n} \\
y_{n}
\end{array}\right]=P\left[\begin{array}{l}
u_{n} \\
v_{n}
\end{array}\right]=\left[\begin{array}{c}
u_{n}+v_{n} \\
\bar{\lambda} u_{n}+\lambda v_{n}
\end{array}\right]
$$

transforms system (9) into

$$
\begin{aligned}
& u_{n+1}=\lambda u_{n}+\sigma\left(\left(u_{n}+v_{n}\right)^{2}+\frac{2-E^{2}}{E^{2}}\left(u_{n}+v_{n}\right)^{3}\right)+O_{4}, \\
& v_{n+1}=\bar{\lambda} v_{n}+\bar{\sigma}\left(\left(u_{n}+v_{n}\right)^{2}+\frac{2-E^{2}}{E^{2}}\left(u_{n}+v_{n}\right)^{3}\right)+O_{4},
\end{aligned}
$$

where

$$
\begin{aligned}
\sigma & =\frac{\lambda}{\lambda-\bar{\lambda}} \cdot \frac{9(E-1)(E+1)}{2 E^{4}}=\frac{\frac{3 \alpha E+i \sqrt{4-9 \alpha^{2} E^{2}}}{2}}{i \sqrt{4-9 \alpha^{2} E^{2}}} \cdot \frac{9 \alpha}{2 E} \\
& =\frac{9 \alpha}{4 E \sqrt{4-9 E^{2} \alpha^{2}}}\left(\sqrt{4-9 E^{2} \alpha^{2}}-3 i \alpha E\right) .
\end{aligned}
$$

Second transformation:

The objective of second transformation is to obtain the nonlinear terms up to order $l-1$ in normal form. The change of variables

$$
\begin{aligned}
& u_{n}=\xi_{n}+\sum_{k=0}^{2}\left(a_{2 k} \xi_{n}^{2-k} \eta_{n}^{k}\right)+\sum_{k=0}^{3}\left(a_{3 k} \xi_{n}^{3-k} \eta_{n}^{k}\right) \\
& v_{n}=\eta_{n}+\sum_{k=0}^{2}\left(\overline{a_{2 k}} \xi_{n}^{k} \eta_{n}^{2-k}\right)+\sum_{k=0}^{3}\left(\overline{a_{3 k}} \xi_{n}^{k} \eta_{n}^{3-k}\right)
\end{aligned}
$$

where

$$
\begin{gathered}
a_{20}=\frac{\sigma}{\lambda(\lambda-1)}, \quad a_{21}=\frac{2 \sigma}{1-\lambda}, \quad a_{22}=\frac{\sigma}{\bar{\lambda}^{2}-\lambda} \\
a_{20}=\frac{\frac{9 \alpha}{E \sqrt{4-9 E^{2} \alpha^{2}}}\left(-\sqrt{-9 E^{2} \alpha^{2}+4}-i(3 E \alpha-2)\right)}{-4(3 E \alpha-2)} \\
a_{21}=\frac{\frac{9 \alpha}{E \sqrt{4-9 E^{2} \alpha^{2}}}}{-2(3 E \alpha-2)}\left(\sqrt{-9 E^{2} \alpha^{2}+4}-i(3 E \alpha-2)\right) \\
a_{22}=\frac{\frac{9 \alpha}{2 E \sqrt{4-9 E^{2} \alpha^{2}}}\left(\sqrt{-9 E^{2} \alpha^{2}+4}(3 E \alpha-1)-3 i(3 E \alpha+1)\left(E \alpha-\frac{2}{3}\right)\right)}{-2(3 E \alpha-2)(3 E \alpha+1)}, \\
a_{20}+\overline{a_{22}}=\frac{9 \alpha}{2 E(3 E \alpha-2)(3 E \alpha+1)}, \\
a_{21}+\overline{a_{21}}=\frac{9 \alpha}{(2-3 E \alpha) E},
\end{gathered}
$$


reduces system (10) to the form

$$
\begin{aligned}
\xi_{n+1} & =\left(\lambda \xi_{n}+\alpha_{2} \xi_{n}^{2} \eta_{n}\right)+O_{4}, \\
\eta_{n+1} & =\left(\bar{\lambda} \eta_{n}+\overline{\alpha_{2}} \xi_{n} \eta_{n}^{2}\right)+O_{4},
\end{aligned}
$$

where

$$
\alpha_{2}=2\left(a_{21}+\overline{a_{21}}\right) \sigma+2\left(a_{20}+\overline{a_{22}}\right) \sigma+(1-2 \alpha) \sigma .
$$

By using that $\alpha=\frac{E^{2}-1}{E^{3}}$, we obtain

$$
\begin{aligned}
\operatorname{Re}\left(\alpha_{2}\right) & =\left(\frac{18 \alpha}{(2-3 \alpha E) E}+\frac{9 \alpha}{E(3 \alpha E-2)(3 \alpha E+1)}+\frac{2-E^{2}}{E^{2}}\right)\left(\frac{9 \alpha}{4 E}\right) \\
& =\frac{-9(E-1)(E+1)\left(2 E^{6}+20 E^{4}-39 E^{2}+18\right)}{2 E^{6}\left(4 E^{2}-3\right)\left(E^{2}-3\right)} .
\end{aligned}
$$

Third transformation:

The change of variables

$$
\begin{aligned}
\xi_{n} & =s_{n}+i t_{n}, \\
\eta_{n} & =s_{n}-i t_{n},
\end{aligned}
$$

transforms system (13) into

$$
\begin{aligned}
& s_{n+1}=\mu_{1} s_{n}-\mu_{2} t_{n}+O_{4}, \\
& t_{n+1}=\mu_{2} s_{n}-\mu_{1} t_{n}+O_{4},
\end{aligned}
$$

where

$$
\begin{aligned}
& \mu_{1}=\operatorname{Re}(\lambda)+\operatorname{Re}\left(\alpha_{2}\right)\left(s_{n}^{2}+t_{n}^{2}\right), \\
& \mu_{2}=\operatorname{Im}(\lambda)+\operatorname{Im}\left(\alpha_{2}\right)\left(s_{n}^{2}+t_{n}^{2}\right) .
\end{aligned}
$$

Since (14) can be write in the form

$$
\begin{aligned}
& s_{n+1}=\cos \omega s_{n}-\sin \omega t_{n}+O_{4}, \\
& t_{n+1}=\sin \omega s_{n}+\cos \omega t_{n}+O_{4},
\end{aligned}
$$

where

$$
\omega=\gamma_{0}+\gamma_{1}\left(s_{n}^{2}+t_{n}^{2}\right)
$$

then for the twist coefficients $\gamma_{0}$ and $\gamma_{1}$ we obtain

$$
\cos \gamma_{0}=\operatorname{Re}(\lambda)=\frac{3 \alpha E}{2}=\frac{3(E-1)(E+1)}{2 E^{2}} \in(0,1) \text { and } \gamma_{1}=-\frac{\operatorname{Re}\left(\alpha_{2}\right)}{\sin \gamma_{0}}
$$

System (15) is the Bikhoff Normal Form for system (9). Since $1<E<\frac{2}{3 \alpha}$ implies

$$
\begin{aligned}
2 E^{6}+20 E^{4}-39 E^{2}+18 & =\left(20 E^{4}-40 E^{2}+20\right)+E^{2}+2\left(E^{6}-1\right) \\
& =20(E-1)^{2}(E+1)^{2}+E^{2}+2\left(E^{6}-1\right)>0,
\end{aligned}
$$

we obtain that $\gamma_{1} \neq 0$. Therefore, the polynomial

$$
\alpha\left(|z|^{2}\right)=\gamma_{0}+\gamma_{1}|z|^{2}=\omega
$$



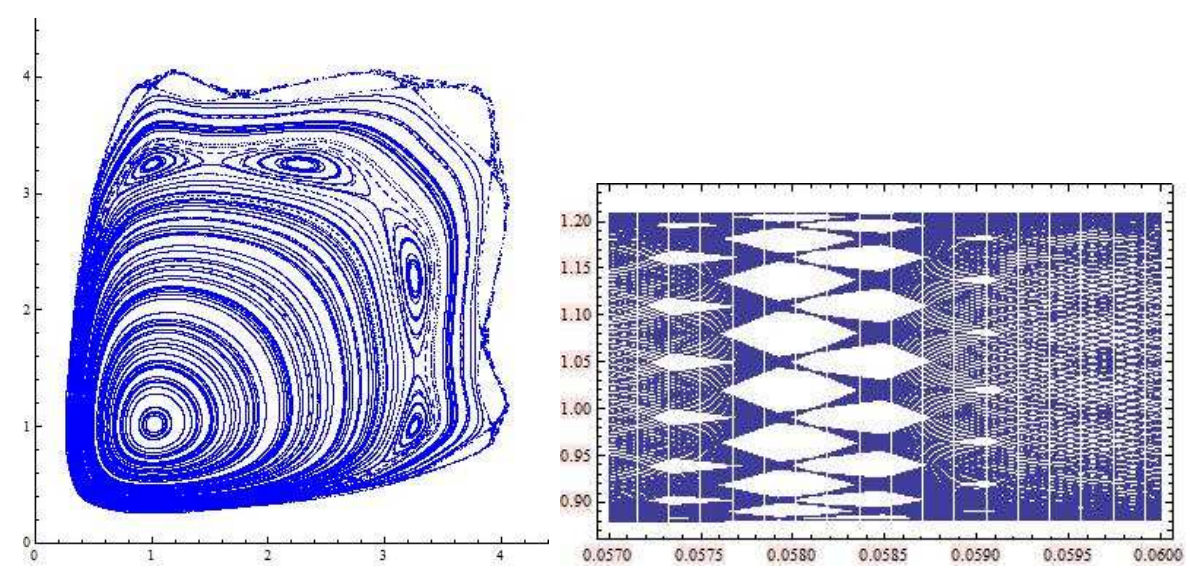

Figure 1. Some orbits of the map $T$ for $\alpha=1 / 27$ and a bifurcation diagram in $(\alpha-x)$-plane. The plots are generated by Dynamica 3 [11].

is not identically zero at the origin and by KAM theorem (Theorem 2) equilibrium point $E=E_{1}$ is stable for $\alpha \in\left(0, \frac{2}{3 \sqrt{3}}\right)$.

\begin{tabular}{|c|c|}
\hline $\bar{P}$ & Solution \\
\hline$\overline{5}$ & 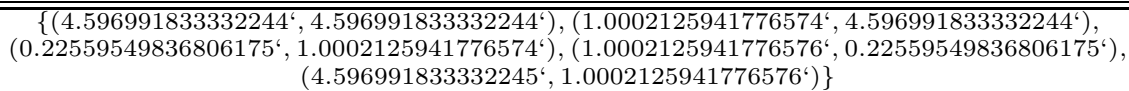 \\
\hline 5 & 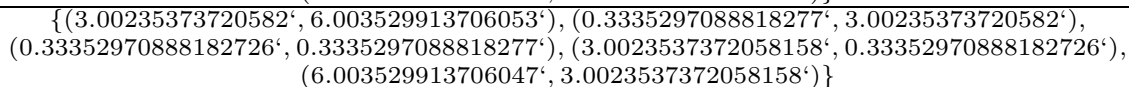 \\
\hline 8 & 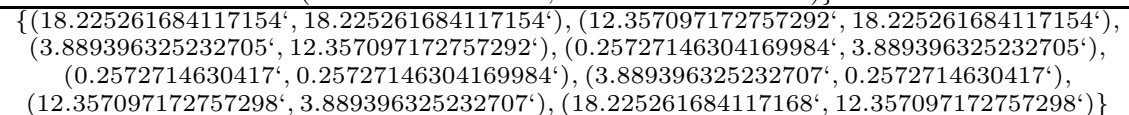 \\
\hline 16 & 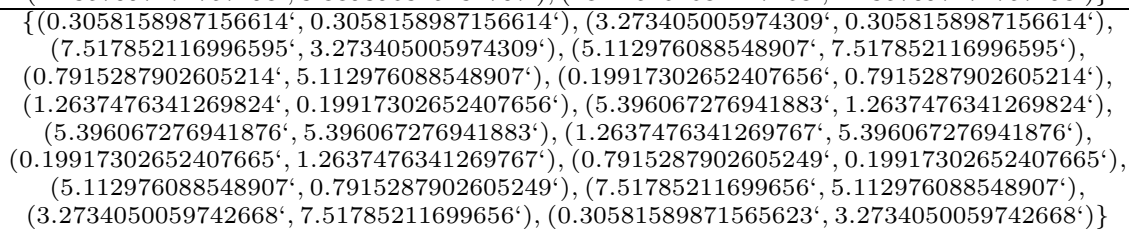 \\
\hline
\end{tabular}

TABLE 1. Some periodic solutions for $\alpha=1 / 27$.

Remark 2. Also, the following result is based on Moser's twist map theorem: There exist periodic points with arbitrarily large periods in every neighborhood of $E=E_{1}$. As in $[4,5,7]$ we see that the eigenvalues $\lambda$ and $\bar{\lambda}$ at the elliptic equilibrium point $E$ are of the form $\lambda=e^{ \pm i \theta}$, where 
$0<\theta<\frac{\pi}{2}$ and that the period of the motion around the equilibrium point must be $q>\frac{2 \pi}{\theta}>4$. For example, if $\alpha=\frac{1}{27}$, then $\frac{2 \pi}{\theta} \approx 4.1498$ so the minimal possible period for a periodic orbit in a neighborhood of the elliptic equilibrium point is 5 .

\section{Symmetries}

In this section, using symmetries for the map $T$, we will show that this map is conjugate to its inverse through an involution. Also, we will use the time reversal symmetry method based on symmetries to effectively find some feasible periods and corresponding orbits of the map $T$.

As we can see in $[3,4,5,7]$, symmetries are important in the study of area-preserving maps because they yield special dynamic behavior. A transformation $R$ of the plane is a time reversal symmetry for $T$ if $R^{-1} \circ T \circ$ $R=T^{-1}$. If time reversal symmetry $R$ satisfies $R^{2}=i d$, then the condition $R^{-1} \circ T \circ R=T^{-1}$ is equivalent to $R \circ T \circ R=T^{-1}$, and then $T$ we can be write as the composition of two involutions $T=I_{1} \circ I_{0}$, where $I_{0}=R$ and $I_{1}=T \circ R$. Note that if $I_{0}=R$ is a reversor, then so is $I_{1}=T \circ R$. Also, the $j$ th involution, defined as $I_{j}:=T^{j} \circ R$, is also a reversor.

The sets

$$
S_{j}=\left\{(x, y) \mid I_{j}(x, y)=(x, y)\right\}, \quad j=1,2,
$$

are invariant sets of the involution maps and they are one-dimensional sets. We call them the symmetry lines of the map $T$. The following result is important for the search periodic orbits, see $[2,5]$.

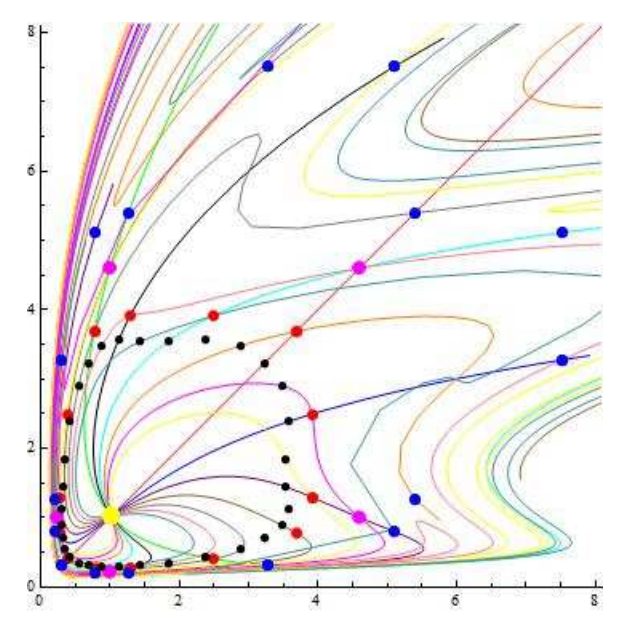

Figure 2. The periodic orbits of period 5 (Magenta), period 14 (Red), period 16 (Blue), period 32 (Black) and E (Yellow) for $\alpha=1 / 27$. The plot is generated by Dynamica 3 [11]. 


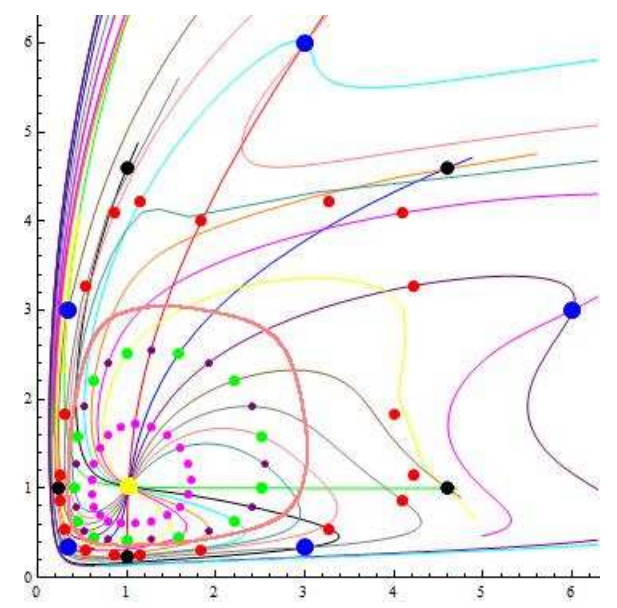

Figure 3. The periodic orbits of period 5 (Blue, Black), period 13 (Green, Purple), 19 (Red), 21 (Magenta), $\infty$ (Pink) and $\mathrm{E}$ (Yellow) for $\alpha=1 / 27$. The plot is generated by Dynamica $3[11]$.

Theorem 6. If $(x, y) \in S_{0,1}$, then $T^{n}(x, y)=(x, y)$ if and only if

$$
\begin{cases}T^{n / 2}(x, y) \in S_{0,1}, & \text { for } n \text { even } \\ T^{(n \pm 1) / 2}(x, y) \in S_{1,0}, & \text { for } n \text { odd }\end{cases}
$$

It means that the periodic orbits of different orders can then be found at the intersection of the symmetry lines $S_{j}, j=1,2, \ldots$ associated to the $j$ th involution, that is, if $(x, y) \in S_{j} \cap S_{k}$, then $T^{j-k}(x, y)=(x, y)$. Also, the symmetry lines satisfy the following relations: $S_{2 j+i}=T^{j}\left(S_{i}\right), \quad S_{2 j-i}=$ $I_{j}\left(S_{i}\right)$, for all $i, j$. For example, for $\alpha=\frac{1}{27}$ in Figure 2, we have an intersection between the symmetry lines $S_{0}$ and $S_{14}=T^{7}\left(S_{0}\right), S_{0}$ and $S_{16}=T^{8}\left(S_{0}\right)$ of the map $T$. The intersection points of these lines correspond to the periodic orbits of period 14 and 16, respectively. Also, see Figure 3 for some examples of the periodic orbits of period 5, 13, 19 and 21, and one orbit which lies on the dense closed curve.

For $\alpha \in\left(0, \frac{2}{3 \sqrt{3}}\right)$ we use the substitution $x_{n}=w_{n}$ and $y_{n}=w_{n-1}$ to transform equation(4) into

$$
\begin{aligned}
& x_{n+1}=\frac{\alpha x_{n}^{3}+1}{y_{n}} \\
& y_{n+1}=x_{n}
\end{aligned}
$$


Then the map $T$ associated to the system (16) is

$$
T(x, y)=\left(\frac{\alpha x^{3}+1}{y}, x\right)
$$

which domain is the positive quadrant $Q$ in $\mathbb{R}^{2}$. The inverse of the map (16) is the map

$$
T^{-1}(x, y)=\left(y, \frac{\alpha y^{3}+1}{x}\right) .
$$

Note that the involution $R(x, y)=(y, x)$ is a reversor for $(16)$ :

$(R \circ T \circ R)(x, y)=(R \circ T)(y, x)=R\left(\frac{\alpha y^{3}+1}{x}, y\right)=\left(y, \frac{\alpha y^{3}+1}{x}\right)=T^{-1}(x, y)$.

So, we have that $T=I_{1} \circ I_{0}$, where $I_{0}(x, y)=R(x, y)=(y, x)$ and

$$
I_{1}(x, y)=(T \circ R)(x, y)=\left(\frac{\alpha y^{3}+1}{x}, y\right) .
$$

The symmetry lines corresponding to $I_{0}$ and $I_{1}$ are

$$
S_{0}=\{(x, y): y=x\}, S_{1}=\left\{(x, y): x=\sqrt{\alpha y^{3}+1}\right\} .
$$

See Figure 2 and Figure 3 for the first twelve iterations of symmetry lines $S_{0}$ and $S_{1}$, respectively, of the map $T$ for $\alpha=\frac{1}{27}$ (for $i=1,2: S_{i}$-Red, $T\left(S_{i}\right)$ Green, $T^{2}\left(S_{i}\right)$-Blue, $T^{3}\left(S_{i}\right)$-Black, $T^{4}\left(S_{i}\right)$-Purple, $T^{5}\left(S_{i}\right)$-Cyan, $T^{6}\left(S_{i}\right)$ Brown, $T^{7}\left(S_{i}\right)$-Orange, $T^{8}\left(S_{i}\right)$-Gray, $T^{9}\left(S_{i}\right)$-Magenta, $T^{10}\left(S_{i}\right)$-Pink, $T^{11}$ $\left(S_{i}\right)$-Yellow and $T^{12}\left(S_{i}\right)$-Dark Red).

Periodic orbits on the symmetry line $S_{0}$ with even period $n$ are searched for by starting with points $\left(x_{0}, x_{0}\right) \in S_{0}$ and imposing that $\left(x_{n / 2}, y_{n / 2}\right) \in S_{0}$, where

$$
\left(x_{n / 2}, y_{n / 2}\right)=T^{n / 2}\left(x_{0}, x_{0}\right) .
$$

This reduces to a one-dimensional root finding for the equation $x_{n / 2}=y_{n / 2}$, where the unknown is $x_{0}$. Also, periodic orbits on $S_{0}$ with odd period $n$ are obtained by solving for $x_{0}$ the equation $x_{(n+1) / 2}^{2}=1+\alpha y_{(n+1) / 2}^{3}$, where

$$
\left(x_{(n+1) / 2}, y_{(n+1) / 2}\right)=T^{(n+1) / 2}\left(x_{0}, x_{0}\right) .
$$

Note that, on analogous way, we can see that the next result is true.

Theorem 7. Assume that $k \geq 3$.

i) If $\alpha>\frac{2}{k}\left(\frac{k-2}{k}\right)^{\frac{k-2}{2}}$, then equation (2) has no positive equilibrium point.

ii) If $\alpha=\frac{2}{k}\left(\frac{k-2}{k}\right)^{\frac{k-2}{2}}$, then equation (2) has a unique positive equilibrium $E=\frac{\sqrt{k(k-2)}}{k-2}$ point which is non-hyperbolic of the parabolic type. 
iii) If $\alpha \in\left(0, \frac{2}{k}\left(\frac{k-2}{k}\right)^{\frac{k-2}{2}}\right)$, then equation (2) has two positive equilibrium points:

a) $E_{1}, 1<E_{1}<\left(\frac{2}{k \alpha}\right)^{\frac{1}{k-2}}$, which is non-hyperbolic of the elliptic type and which is stable,

b) $E_{2}>\left(\frac{2}{k \alpha}\right)^{\frac{1}{k-2}}$, which is a saddle.

\section{REFERENCES}

[1] A. Cima, A. Gasull and V. Manosa, Non-integrability of measure preserving maps via Lie symmetries, J. Differ. Equ., 259 (2015), 5115-5136.

[2] D. del-Castillo-Negrete, J. M. Greene and E. J. Morrison, Area preserving nontwist maps: periodic orbits and transition to chaos, Physica D, 91 (1986), 1-23.

[3] E. Dennete, M. R. S. Kulenović and E. Pilav, Birkhoff normal forms, KAM theory and time reversal symmetry for certain rational map, Mathematics 2016, 4, 20; doi:10.3390/math4010020.

[4] M. Garić-Demirović, M. Nurkanović and Z. Nurkanović, Stability, periodicity and symmetries of certain second order fractional difference equation with quadratic terms via KAM theory, Math. Methods Appl. Sci., 2016, in print.

[5] M. Gidea, J. D. Meiss, I. Ugarcovici and H. Weiss, Applications of KAM theory to population dynamics, J. Biol. Dyn., 5 (1)(2011), 44-63.

[6] J. K. Hale and H. Kocak, Dynamics and Bifurcation, Springer-Verlag, New York, 1991.

[7] S. Jašarević-Hrustić, M. R. S. Kulenović, Z. Nurkanović and E. Pilav, Birkhoff normal forms, KAM theory and siymmetries for certain second order rational difference equation with quadratic term, Int. J. Difference Equ., 10 (2) (2015), 181-199.

[8] E. J. Janowski, M. R. S. Kulenović and Z. Nurkanović, Stability of the $k$-th order Lyness' equation with a period-k coefficient, Int. J. Bifurcation Chaos Appl. Sci. Eng., 17 (2007), 143-152.

[9] V. L. Kocic, G. Ladas, G. Tzanetopoulos and E. Thomas, On the stability of Lyness' equation, Dyn. Contin. Discrete Impulsive Syst., 1 (1995), 245-254.

[10] M. R. S. Kulenović, Invariants and related Liapunov functions for difference equations, Appl. Math. Lett., 13 (2000), 1-8.

[11] M. R. S. Kulenović and O. Merino, Discrete Dynamical Systems and Difference Equations with Mathematica, Chapman and Hall/CRC, Boca Raton, London, 2002.

[12] M. R. S. Kulenović and Z. Nurkanović, Stability of Lyness' equation with period-three coeffcient, Rad. Mat., 12 (2004), 153-161.

[13] M. R. S. Kulenović and Z. Nurkanović, Stability of Lyness' equation with period-two coeficient via KAM theory, J. Concr. Appl. Math., 6 (2008), 229-245.

[14] M. R. S. Kulenović, Z. Nurkanović and E. Pilav, Birkhoff normal forms and KAM theory for Gumowski-Mira equation, The Scientific World Journal, Volume 2014, Article ID 819290, 8 p.

[15] G. Ladas, G. Tzanetopoulos and A. Tovbis, On May's host parasitoid model, J. Difference Equ. Appl., 2 (1996), 195-204.

[16] G. Papaschinopoulos and C. J. Schinas, Stability of a class of nonlinear difference equations, J. Math. Anal. Appl., 230 (1999), 211-222. 
KAM THEORY AND PERODICITY FOR CERTAIN DIFFERENCE EQUATION 231

[17] M. Tabor, Chaos and Integrability in Nonlinear Dynamics. An Introduction, A WileyInterscience Publication, John Wiley and Sons, Inc., New York, 1989.

[18] E. C. Zeeman, Geometric unfolding of a difference equation, Preprint, Hertford College, Oxford (1996).

(Received: May 23, 2016)

Department of Mathematics

University of Tuzla

75000 Tuzla

Bosnia and Herzegovina

mehmed.nurkanovic@untz.ba

zehra.nurkanovic@untz.ba 
